\title{
Auxiliary Liver Graft Can Be Protected From HBV Infection in HBsAg Positive Blood Circulation
}

\author{
Lin Wei ${ }^{1,2,3+}$, Hai-Ming Zhang ${ }^{1,2,3+}$, Chi-Dan Wan ${ }^{4}$, Wei Qu ${ }^{1,2,3}$, Zhi-Gui Zeng ${ }^{1,2,3}$, \\ Ying Liu ${ }^{1,2,3}$, Jun Xiong ${ }^{4}$, Li-Ying Sun ${ }^{1,2,3 *}$ and Zhi-Jun Zhu ${ }^{1,2,3 *}$
}

${ }^{1}$ Beijing Friendship Hospital, Capital Medical University, Beijing, China, ${ }^{2}$ Clinical Center for Pediatric Liver Transplantation, Capital Medical University, Beijing, China, ${ }^{3}$ National Clinical Research Center for Digestive Diseases, Beijing, China, ${ }^{4}$ Department of Hepatobiliary Surgery, Union Hospital, Tongji Medical College, Huazhong University of Science and Technology, Wuhan, China

OPEN ACCESS

Edited by:

Jian Wu,

Zhejiang University, China

Reviewed by:

Dawei Cui,

Zhejiang University, China

Dai Yu Zhu,

The 117th Hospital of PLA, China

${ }^{*}$ Correspondence:

Li-Ying Sun

sunxIx@outlook.com

Zhi-Jun Zhu

zhu-zhijun@outlook.com

tThese authors have contributed equally to this work and share first authorship

Specialty section:

This article was submitted to Infectious Diseases - Surveillance,

Prevention and Treatment,

a section of the journal

Frontiers in Medicine

Received: 17 June 2021 Accepted: 04 August 2021

Published: 25 August 2021

Citation:

Wei L, Zhang H-M, Wan C-D, Qu W, Zeng Z-G, Liu Y, Xiong J, Sun L-Y and

Zhu Z-J (2021) Auxiliary Liver Graft Can Be Protected From HBV Infection in HBsAg Positive Blood Circulation.

Front. Med. 8:726502.

doi: 10.3389/fmed.2021.726502
Auxiliary grafts have a high risk of Hepatitis B virus (HBV) infection in patients with chronic HBV-related diseases. Hepatitis B virus-related auxiliary partial orthotopic liver transplantation (APOLT) cases were reviewed to show the results of current methods to block native-to-graft HBV transmission. Three patients received APOLT for HBV-related liver cirrhosis and a recurrent upper gastrointestinal hemorrhage between April 2015 and January 2017 by the liver transplant team of Beijing Friendship Hospital affiliated with Capital Medical University. All three patients were positive for HBV surface antigen (HBsAg) and had a negative HBV DNA test result before transplantation. After auxiliary transplantations, HBsAg was found to be positive in two patients and negative in one patient. To avoid graft infection of HBV, entecavir-based therapy was employed and the remnant native livers of the recipients were removed 51-878 days after liver transplantation. Then, serum conversions of HBsAg were found in all three cases. For the first time, this case series shows the possibility of blocking the transmission of HBV from a native liver to a graft in auxiliary transplantation by entecavir-based therapy. Among the cases, a left lobe graft was successfully implanted as a replacement of the right lobe of the recipient, which is also discussed.

Keywords: APOLT, entecavir, liver cirrhosis, liver regeneration, hepatitis B recurrence

\section{INTRODUCTION}

In auxiliary partial orthotopic liver transplantation (APOLT), a liver graft is implanted while the partial or entire native liver is preserved. Two livers will co-function and support each other. Auxiliary partial orthotopic liver transplantation is commonly used in patients with acute liver failure or metabolic liver diseases $(1,2)$. Patients who receive APOLT for acute liver failure, a reversible liver disease, may be able to discontinue immunosuppressant treatment after their native liver has regenerated (3). Some inherent liver metabolic defects can be corrected by a small auxiliary graft as a special form of gene therapy $(4,5)$. Auxiliary partial orthotopic liver transplantation can also be employed when a whole liver graft cannot be acquired or the graft volume is inadequate $(6,7)$. 
Hepatitis B virus (HBV)-related diseases are regarded as a contraindication for APOLT. The HBV-infected native liver is preserved in APOLT, which may potentially relieve $\mathrm{HBV}$ and increase the risk of graft infection. Hepatitis B virus recurrence after liver transplantation may progress rapidly and become difficult to control. Several reports showed attempts to carry out auxiliary liver transplantations in patients with HBV-related liver diseases. In the report of Onno, in 1988, four cases received a heterotopic liver transplantation for HBV-related liver cirrhosis, and immunofluorescence stains for the core antigen were positive in all of the grafts within 3 weeks after transplantation (8). One of these patients had clinical signs (recurrence of ascites). In 1991 and 1992, Kate FJ reported chronic hepatitis and cirrhosis in the auxiliary graft after auxiliary liver transplantation for HBV related liver diseases $(9,10)$. In the auxiliary liver transplantations for fulminant hepatitis B reported by Durand in 2002 (11), three patients who were positive for $\mathrm{HBV}$ surface antigen (HBsAg) received an auxiliary liver transplantation. Two patients survived, and one was followed up more than 1 year later. HBV surface antigen was found to be negative in these patients after the hepatitis B immune globulin (HBIG) treatment. However, in the only patient followed for more than 1 year, the HBIG titer was negative, which was most likely a residue of HBV. In 2008, Quaglia reported auxiliary liver transplantations for seronegative liver failure (12). Four cases of HBV infections were included. One of these patients died of HBV recurrence on day 1,505, and the other three patients lived (12). According to the results of these cases, the auxiliary graft conveyed a high risk of $\mathrm{HBV}$ infection in patients with chronic HBV-related diseases.

Great improvements in anti-HBV agents have been made. Nucleos(t)ide analogs and HBIG have been used as a combined therapy to prevent $\mathrm{HBV}$ recurrence after liver transplantation, which significantly reduces the $\mathrm{HBV}$ recurrence rate. In a report by Wang in 2017 (13), four patients with HBV-related liver cirrhosis received APOLT. Nucleos(t)ide analogs were administered after APOLT. After transplantation, all recipients were positive for serum $\mathrm{HBsAg}$ and negative for the $\mathrm{HBsAg}$ and $\mathrm{HBV}$ core antigen $(\mathrm{HBcAg})$ in grafts. All patients lived and showed normal graft function within a mean follow-up period of 21 (13-26) months. However, immunostains for HBsAg and $\mathrm{HBcAg}$ at 1,6 , and 12 months after transplantation were not enough to exclude HBV infection in the auxiliary grafts. Whether the nucleos(t)ide analog blocked HBV infection should be further confirmed.

Because of insufficient donor graft volumes, three patients received APOLTs for HBV-related liver cirrhosis in our center. To avoid HBV infection of the auxiliary grafts, we removed the remnant native livers of the recipients 51-878 days after the liver transplantation. Then, serum HBsAg became negative in all three

Abbreviations: APOLT, auxiliary partial orthotopic liver transplantation; HBV, Hepatitis B virus; HBsAg, HBV surface antigen; HBIG, Hepatitis B immune globulin; $\mathrm{HBcAg}, \mathrm{HBV}$ core antigen; GRWR, graft-to-recipient weight ratio; SFSS, small for size syndrome; HBsAb, HBV surface antibody; ICU, intensive care unit; ALT, alanine aminotransferase; AST, aspartate aminotransferase; TBIL, total bilirubin; MRCP, magnetic resonance cholangiopancreatography; PTCD, percutaneous transhepatic cholangiodrainage; $\mathrm{HBeAg}, \mathrm{HBV}$ e antigen; $\mathrm{HBeAb}$, HBV e antibody; Pre-S1Ag, pre S1 antigen; PV, portal vein. recipients. For the first time, this small case series shows that it is possible to completely block the transmission of HBV from the native liver to a graft in auxiliary transplantation by entecavir monotherapy or in combination with HBIG therapy.

\section{PATIENTS AND METHODS}

Three patients who received APOLTs for HBV-related liver cirrhosis were reviewed; These APOLTs were conducted by the liver transplant team of Beijing Friendship Hospital affiliated with Capital Medical University. This study and the APOLT treatment were approved by the ethics committee of Beijing Friendship Hospital. Informed consent for the operations and this study was obtained. All three patients received APOLT for HBV-related liver cirrhosis and recurrent upper gastrointestinal hemorrhage. Living donor liver transplantation was the only option for these patients due to the shortage of organs (14). However, the left lobe of each donor liver was too small to be transplanted, with a graftto-recipient weight ratio (GRWR) less than $0.8 \%$. Additionally, the right lobe could not be donated because the volume of the remnant liver was insufficient to ensure the donor's safety because the proportion was $<35 \%$ (Table 1). Thus APOLT was employed to prevent small for size syndrome (SFSS).

The characteristics of the donors and recipients are provided in Table 2. Patient A was 55 years old and was admitted for a recurrent upper gastrointestinal hemorrhage. His first upper gastrointestinal hemorrhage was found on January $6^{\text {th }}, 2015$, and then, splenectomy and disconnection were performed in 2007. Patient C, a 52-year-old man, was admitted with the same diagnosis and no surgical history. Both of these patients received left lobe APOLT by replacing the left lobes of their native livers on April 26 ${ }^{\text {th }}, 2015$, and January $11^{\text {th }}, 2017$, respectively. Patient B was 29 years old. The patient experienced an upper gastrointestinal hemorrhage in 2010. Splenectomy and disconnection were conducted to prevent recurrent hemorrhage. In November 2011, the patient suffered from a severe upper gastrointestinal hemorrhage again. Although endoscopic sclerotherapy was performed, it did not stop recurrent hemorrhage. APOLT was planned. Abdominal adhesions due to previous surgery limited the space of the upper left abdominal cavity. Finally, we implanted the left lobe graft into the upper right abdominal cavity of the recipient after removing the right lobe of his native liver on August $16^{\text {th }}, 2016$ (Figure 1).

Left lobes without the middle hepatic vein were procured as grafts in all of these APOLTs. In patient A, the left hepatic vein, left portal vein and left hepatic artery were anastomosed to their corresponding parts of the recipient. In patient $\mathrm{B}$, the left hepatic vein, left portal vein, and left hepatic artery were anastomosed to the right hepatic vein, right portal vein, and right hepatic artery of the recipient. The left hepatic duct was reconstructed by biliary-enterostomy in patient $\mathrm{B}$. In patient $\mathrm{C}$, the portal vein (PV) of the graft was elongated by a vein conduit taken from the left portal vein of the recipient and anatomized to the recipient's PV by the end to side method. The left hepatic artery of the graft was reconstructed to the left hepatic artery of the recipient. An auxiliary left hepatic artery was also found in patient $\mathrm{C}$. 
TABLE 1 | Volumetric assessment of left/right lobe of living donor by computerized tomography.

\begin{tabular}{|c|c|c|c|c|c|c|}
\hline Donor and patient & Potential graft & Volume of graft (ml) & RLV/WLV (\%) & Body weight of recipient $(\mathrm{kg})$ & GRWR (\%) & Conclusion \\
\hline \multirow[t]{2}{*}{ Donor of patient $A$} & Left lobe & 396 & 69.9 & 68 & 0.58 & Insufficient graft \\
\hline & Right lobe & 921 & 30.1 & & 1.35 & Insufficient remnant liver \\
\hline \multirow[t]{2}{*}{ Donor of Patient B } & Left lobe & 448 & 68.0 & 76 & 0.59 & Insufficient graft \\
\hline & Right lobe & 952 & 32.0 & 76 & 1.25 & Insufficient remnant liver \\
\hline \multirow[t]{2}{*}{ Donor of Patient C } & Left lobe & 390 & 72.7 & 73 & 0.53 & Insufficient graft \\
\hline & Right lobe & 1,041 & 27.3 & 73 & 1.42 & Insufficient remnant liver \\
\hline
\end{tabular}

RLV, remnant liver volume; WLV, whole liver volume; GRWR, graft recipient's body weight ratio.

TABLE 2 | Characteristics of donors and recipients in auxiliary liver transplantation.

\begin{tabular}{|c|c|c|c|}
\hline & Patient A & Patient B & Patient C \\
\hline \multicolumn{4}{|l|}{ Recipient } \\
\hline Age (year)/sex & 55/male & 29/male & 52/male \\
\hline Cause of liver cirrhosis & Hepatitis B & Hepatitis B & Hepatitis B \\
\hline ABO blood group & A & $\mathrm{B}$ & O \\
\hline Serum result of HBV & $\begin{array}{l}\operatorname{HBsAg}(+) \\
\operatorname{HBsAb}(-) \\
\operatorname{HBeAg}(-) \\
\operatorname{HBeAb}(-) \\
\operatorname{HBcAb}(+)\end{array}$ & $\begin{array}{l}\operatorname{HBsAg}(+) \\
\operatorname{HBsAb}(-) \\
\operatorname{HBeAg}(-) \\
\operatorname{HBeAb}(+) \\
\operatorname{HBcAb}(+)\end{array}$ & $\begin{array}{l}\operatorname{HBsAg}(+) \\
\operatorname{HBsAb}(-) \\
\operatorname{HBeAg}(-) \\
\operatorname{HBeAb}(+) \\
\operatorname{HBcAb}(+)\end{array}$ \\
\hline Child grade & 6 & 6 & 7 \\
\hline MELD score & 11 & 9 & 11 \\
\hline Platelet count & $102 \times 10^{9} / \mathrm{L}$ & $382 \times 10^{9} / \mathrm{L}$ & $45 \times 10^{9} / L$ \\
\hline Albumin (g/L) & 33.3 & 38.0 & 43.1 \\
\hline Total bilirubin ( $\mu \mathrm{mol} / \mathrm{L})$ & 22.0 & 18.87 & 37.96 \\
\hline INR & 1.43 & 1.18 & 1.18 \\
\hline Creatinine (Imol/L) & 66.0 & 67.5 & 73.1 \\
\hline Serum sodium (Imol/L) & 138.9 & 139 & 140.8 \\
\hline Ascites & No & No & mild \\
\hline Esophageal and gastric varices & Severe & Severe & Severe \\
\hline \multicolumn{4}{|l|}{ Donor } \\
\hline Age (year)/sex & 45/female & 50/male & 47/female \\
\hline Serum result of HBV & $\begin{array}{l}\operatorname{HBsAg}(-) \\
\operatorname{HBsAb}(+) \\
\operatorname{HBeAg}(-) \\
\operatorname{HBeAb}(-) \\
\operatorname{HBcAb}(+)\end{array}$ & $\begin{array}{l}\operatorname{HBsAg}(-) \\
\operatorname{HBsAb}(+) \\
\operatorname{HBeAg}(-) \\
\operatorname{HBeAb}(-) \\
\operatorname{HBcAb}(-)\end{array}$ & $\begin{array}{l}\operatorname{HBsAg}(-) \\
\operatorname{HBsAb}(+) \\
\operatorname{HBeAg}(-) \\
\operatorname{HBeAb}(-) \\
\operatorname{HBcAb}(-)\end{array}$ \\
\hline \multicolumn{4}{|l|}{ Graft } \\
\hline Type of graft (lobe) & $\begin{array}{l}\text { Left lobe (without middle } \\
\text { hepatic vein) }\end{array}$ & $\begin{array}{l}\text { Left lobe (without middle } \\
\text { hepatic vein) }\end{array}$ & $\begin{array}{l}\text { Left lobe (without middle } \\
\text { hepatic vein) }\end{array}$ \\
\hline Graft weight during operation (g) & 386 & 408 & 349 \\
\hline ABO blood group & A & $\mathrm{O}$ & $\mathrm{O}$ \\
\hline Cold ischemic time & $2 \mathrm{~h} 5 \min$ & $6 \mathrm{~h} 15 \mathrm{~min}$ & 4h $10 \mathrm{~min}$ \\
\hline Warm ischemic time & $1 \mathrm{~min}$ & $1 \mathrm{~min}$ & $1 \mathrm{~min}$ \\
\hline GW/RW & $0.57 \%$ & $0.54 \%$ & $0.46 \%$ \\
\hline Duration of operation & $9 \mathrm{~h} 36 \mathrm{~min}$ & $13 \mathrm{~h} 50 \mathrm{~min}$ & $12 \mathrm{~h} 20 \mathrm{~min}$ \\
\hline Blood loss (ml) & 200 & 3,000 & 2,400 \\
\hline Post-operative complications & Anatomotic stricture & None & Anatomotic stricture \\
\hline Follow-up (days) & 1,200 & 566 & 525 \\
\hline
\end{tabular}




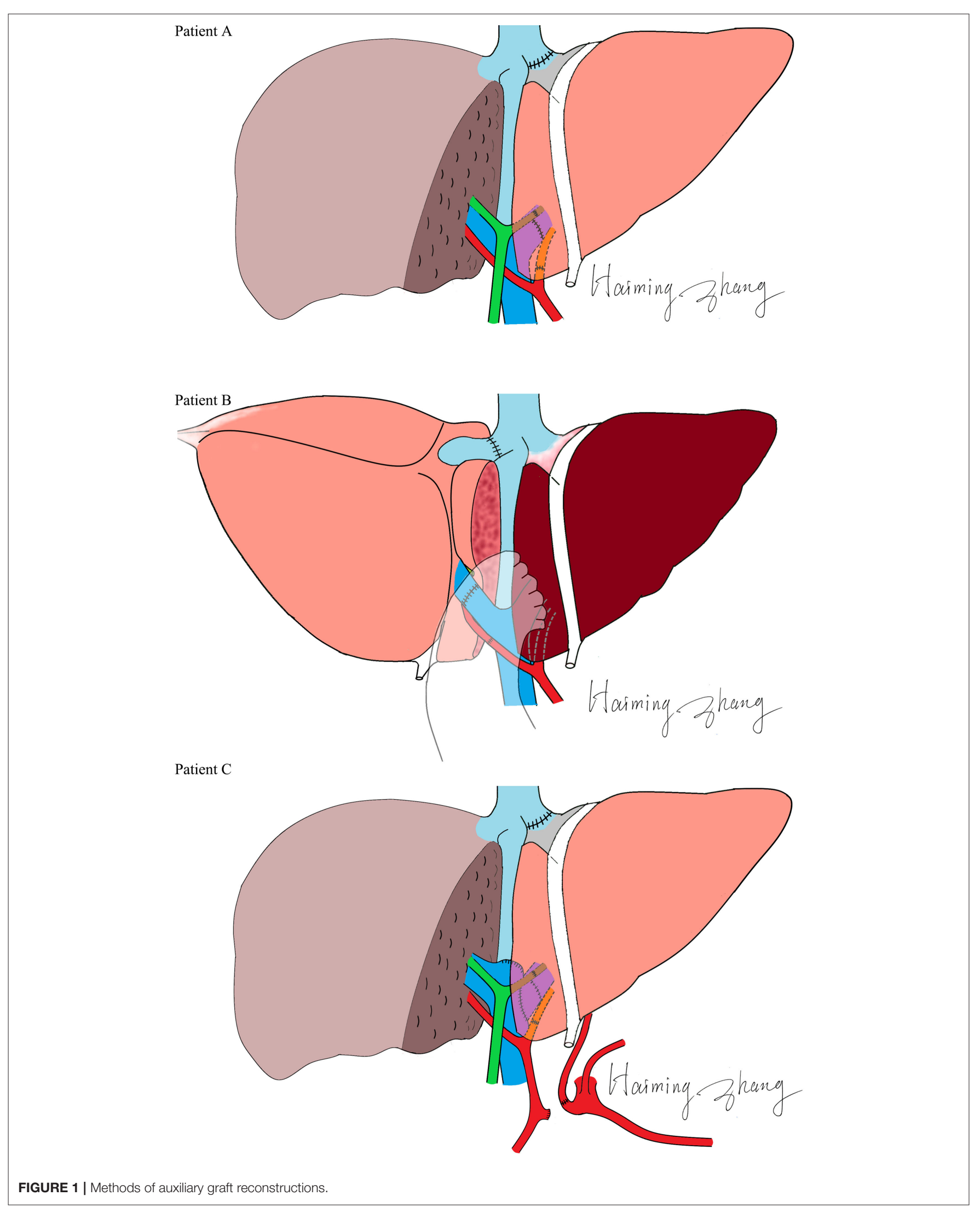


The common hepatic artery of patient $\mathrm{C}$ was transected. The proximal end was connected to the auxiliary left hepatic artery of the graft, and the distal end was closed. End to end biliary anastomoses were performed in patients $\mathrm{A}$ and $\mathrm{C}$.

After APOLT, the biochemistry results showed normal liver function in all three patients. The volumes of the grafts increased over time, and atrophy was found in the remnant native livers. We removed the remnant native livers at 51-878 days after transplantation to prevent $\mathrm{HBV}$ infection (patients $\mathrm{A}, \mathrm{B}$, and $\mathrm{C}$ at 878,136 , and 51 days, respectively).

Antibiotics, immunosuppressant, and antithrombotic agents were administered. Tacrolimus, mycophenolate mofetil, and steroids were included in the immunosuppression regimen. The target serum level of tacrolimus was 6 and $8 \mathrm{ng} / \mathrm{ml}$. Entecavir was administered continuously since APOLT. Hepatitis B immune globulin was not given to patient $\mathrm{A}$. Patient $\mathrm{B}$ and patient $\mathrm{C}$ received 4,000 IU HBIG during APOLT and 2,000-4000 IU HBIG once per day within the first week after transplantation. If the patient was positive for the HBV surface antibody (HBsAb), HBIG was given according to the blood titer. Hepatitis B immune globulin therapy was not continued if a patient was persistently positive for $\mathrm{HBsAg}$ and negative for $\mathrm{HBsAb}$. In patient $\mathrm{B}$ and patient $\mathrm{C}, 4,000 \mathrm{IU} \mathrm{HBIG}$ was also given during the hepatectomy, and then, HBIG was administered based on the blood titer.

Routine blood tests, biochemical markers for liver and kidney functions, and ultrasonography were conducted at the early stages, after transplantation and at each follow-up. Computed tomography (CT) was used to estimate the volumes of the grafts and native livers, if necessary. Liver biopsies were performed during the hepatectomies.

\section{RESULTS}

\section{Graft Function and Complications}

All three patients recovered from the operations, and their liver functions were normal at the end of the follow-up. The duration of the intensive care unit (ICU) stay was 3, 2 and 2 days for patients A, B, and C, respectively. No serious infections or surgical complications were found. Antibiotics were stopped 5-7 days after transplantation. Patients were discharged 2128 days after transplantation. Patient A showed elevated levels of alanine aminotransferase (ALT), aspartate aminotransferase (AST), and total bilirubin (TBIL) 136 days after transplantation. Magnetic resonance cholangiopancreatography (MRCP) showed a biliary anastomotic stricture, and a percutaneous transhepatic cholangiodrainage (PTCD) was placed. Then, the biochemical parameters returned to normal. However, choledocholithiasis occurred 829 days after transplantation. Choledoenterostomy was performed during the hepatectomy at the $878^{\text {th }}$ day after transplantation. Finally, the biochemical parameters of the liver function became normal. Patient B recovered smoothly; no complications were found during follow-up. A biliary anastomotic stricture was also found in patient C 391 days after transplantation, which was treated successfully by PTCD. No serious infections, acute rejections or chronic rejections were found among these patients. According to the results of ultrasonography, the artery, PV, and hepatic vein of the grafts were patent and the blood flow velocities were within the normal range. The levels of ALT, AST, and TBIL are shown in Figure 2.

\section{HBV Markers}

All recipients were positive for serum HBsAg before liver transplantation. Patient A and patient B were HBsAg positive after transplantation even after receiving entecavir and HBIG therapy. Patient $\mathrm{C}$ showed unstable HBsAg and HBsAb levels after transplantation. All three patients were $\mathrm{HBsAg}$ negative and $\mathrm{HBsAb}$ and $\mathrm{HBV}$ core antibody ( $\mathrm{HBcAb}$ ) positive after hepatectomy (Figure 3). Three hundred and twenty-two days after the complete removal of his native liver, patient $\mathrm{A}$ was negative for all of the serum markers of $\mathrm{HBV}$, including $\mathrm{HBs} A$, HBsAb, HBV e antigen (HBeAg), HBV e antibody (HBeAb), $\mathrm{HBcAb}$, and pre S1 antigen (Pre-S1Ag). Patient B and patient $\mathrm{C}$ were followed for 566 and 525 days, respectively, and no changes in the serum markers of HBV were found since hepatectomies. All three patients were negative for HBV DNA before and after transplantation, which was tested every 2 weeks after transplantation until discharge after hepatectomy. Biopsies were taken from the grafts during hepatectomy. Immunostaining of donor liver tissues for $\mathrm{HBsAg}$ and $\mathrm{HBcAg}$ were all negative.

\section{Graft Volume}

Computed tomography data of patients could be found at several time points. We calculated volumes of grafts and native livers by the available Computed tomography data and showed them in Figure 4. The volumes of the grafts increased over time, especially in the early stages after liver transplantation (Figure 4). Since the remnant native livers were cirrhotic, the blood supply to them was very low, which was detected by ultrasonography but could not be quantified. The volume of the remnant native liver continued to reduce as a result of portal blood competition with the graft (Figure 4). The trend of the PV blood velocity changes was roughly matched with the total volume changes of grafts. However, the PV blood velocities varied greatly at the early stages after transplantation, and the low density of examinations at the late stages cannot show a clear trend of PV blood velocity changes.

\section{DISCUSSION}

In these three APOLT recipients, the remnant native livers releasing $\mathrm{HBsAg}$ may be potential sources of $\mathrm{HBV}$. However, the liver grafts were not infected, which can be supported by HBsAg conversions after removing native livers. Thus, in these APOLTs, entecavir completely blocked HBV transmission from the HBV-infected native livers to the grafts, even though the two partial livers shared blood circulation for 51 to 878 days. The combination of lamivudine plus HBIG is the most widely used treatment to prevent $\mathrm{HBV}$ recurrence after liver transplantation for HBV-related diseases. Lamivudine alone has been shown to be insufficient to completely block the transmission of $\mathrm{HBV}$ to the HBV-free graft after liver transplantation (15). New nucleos(t)ide analogs have been used and showed high efficacy in preventing $\mathrm{HBV}$ recurrence (16). Entecavir is also used as monotherapy to prevent $\mathrm{HBV}$ recurrence (17). However, 

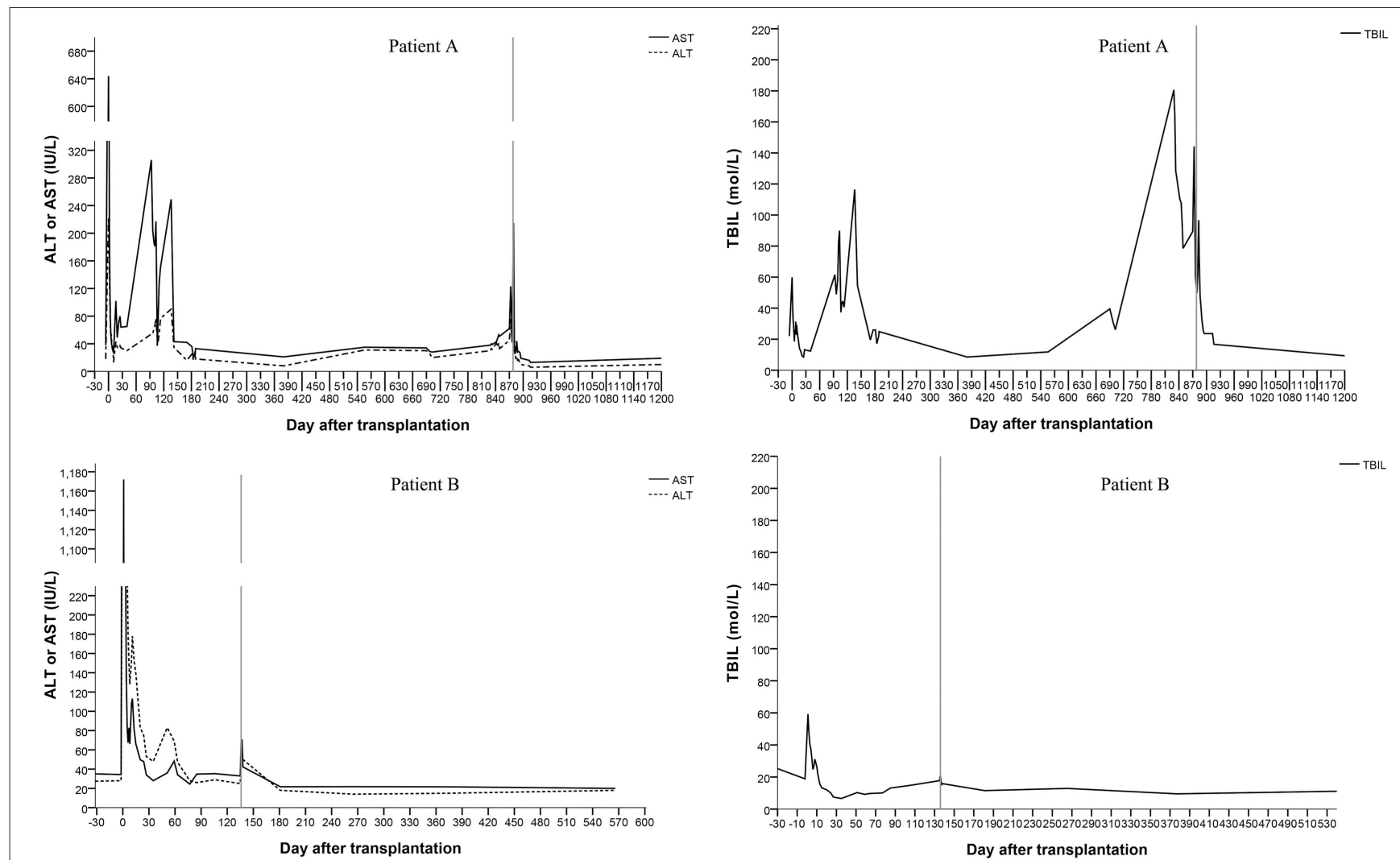

Day after transplantation
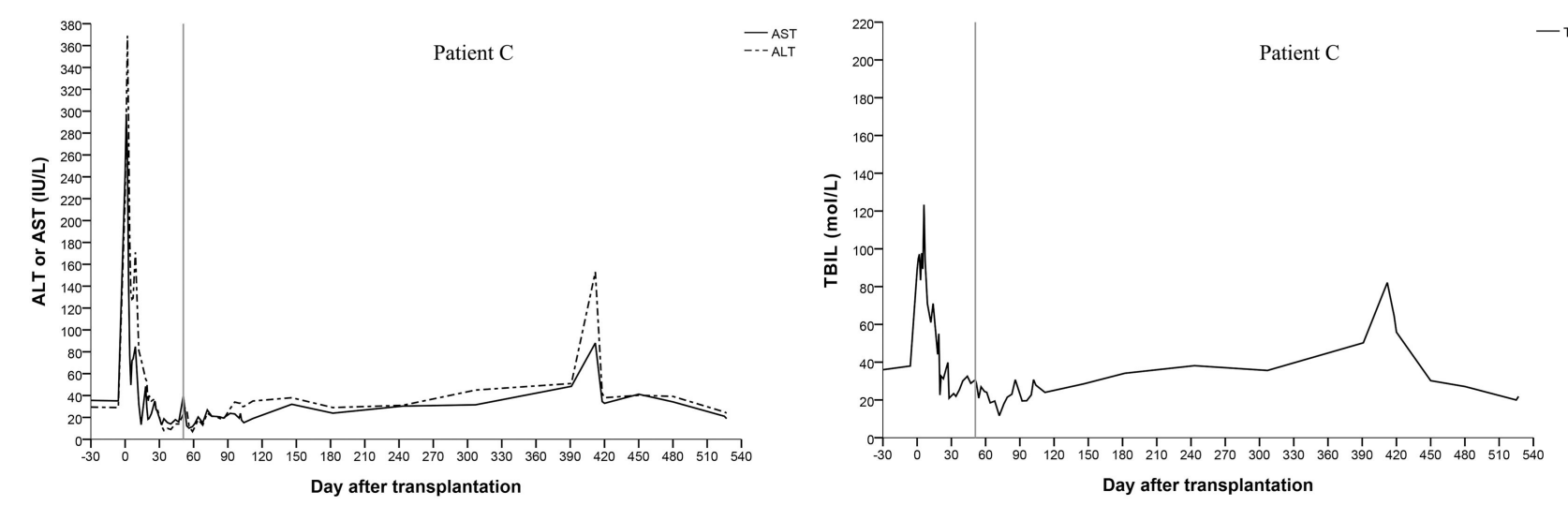

FIGURE 2 | Changes of the serum biochemical parameters for liver function (the vertical line shows the date of the hepatectomy of the remnant native liver).

it is not clear whether HBV transmission can be completely blocked by nucleo(t)ide analogs in HBV-related APOLT. In classic liver transplantation, the HBV infected liver is completely removed. Thus, entecavir can block HBV infection after the serum conversion of HBsAg. In auxiliary liver transplantation, part of the HBV-infected liver continues to release HBsAg. The auxiliary graft seems to inevitably become infected by HBV if entecavir cannot completely prevent viron formation. Liver transplant recipients are immuno-compromised, which also increases the risk of HBV infection. Previous clinical studies (see the introduction) of HBV-related auxiliary liver transplantation showed that auxiliary grafts were normal in function and negative in immunological stains for HBsAg and HBcAg after entecavir administration, which suggested the practical of APOLT for HBV related diseases. Our cases further demonstrated the possibility of preventing graft infection of $\mathrm{HBV}$ in auxiliary liver transplantation, especially in patient A whose native liver was removed 878 days after transplantation. By removing the remnant native liver, HBV infection can be cured after APLOT. Keeping grafts free from HBV infection should be emphasized in the process of auxiliary liver transplantation. In the current series, only patients with liver cirrhosis received APOLT. The blood flow resistance in the cirrhotic native liver increased dramatically compared to that in the grafts. Thus, portal blood 

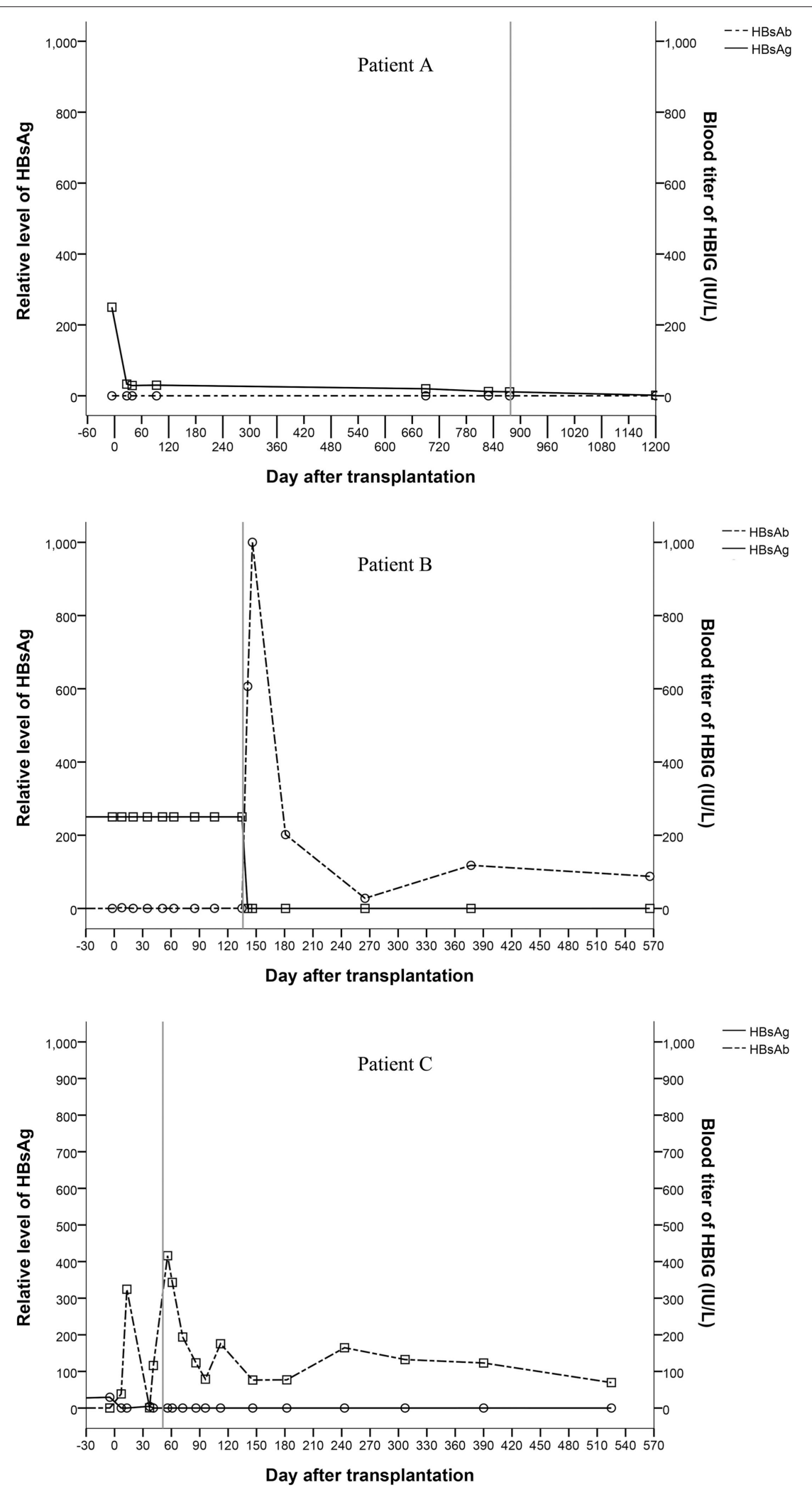

FIGURE 3 | Changes of serum markers for HBV (the vertical line shows the date of the hepatectomy of the remnant native liver). 

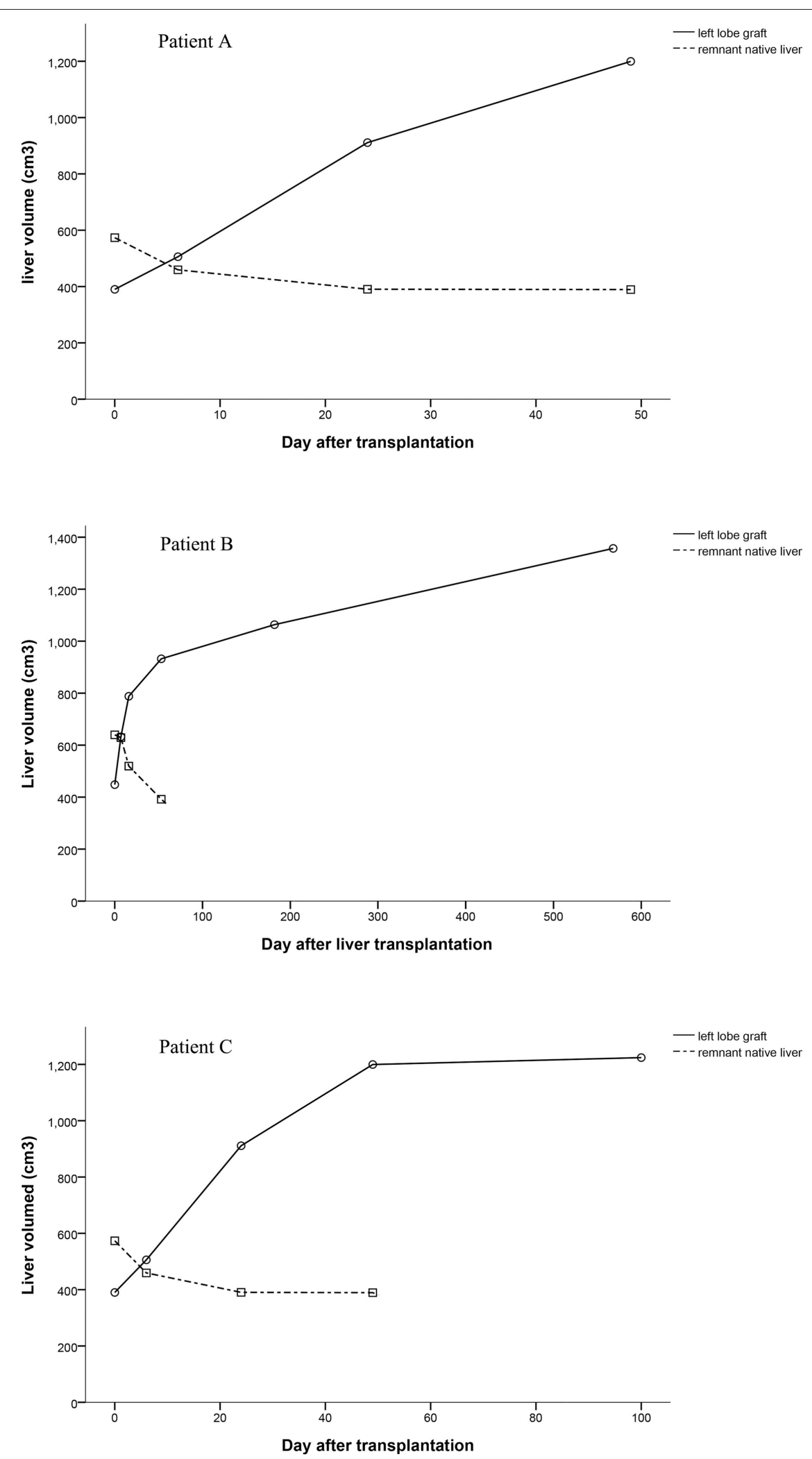

FIGURE 4 | Volume changes of the auxiliary grafts and native liver. 
flow of the native livers was too low to be quantified, and most of the portal blood supply went to the grafts. Fast increases in the graft volumes were found, together with atrophy of the native livers (Figure 4). In functional respects, the remnant native livers contributed little to the total liver function and could be safety removed at relative early stages. Therefore, we removed the remnant native liver of patient $\mathrm{C}$ only 51 days after transplantation to reduce the graft exposure to $\mathrm{HBsAg}$ positive circulation. Thus, effective anti-HBV treatment and early removal of the remnant liver are suggested in APOLT for $\mathrm{HBV}$-related liver cirrhosis to reduce the risk of $\mathrm{HBV}$ infecting grafts.

A special manner of left lobe graft implantation was used in patient B. The left lobe graft was implanted instead of the right lobe of the recipient. This successful case suggested the practicability of this operation manner, which may be a promising way to settle the space issue in left lobe implantation. Abdominal adhesions resulting from previous surgeries are a common problem, as splenectomies with disconnection are conventional treatments. By reconstructing the normal anatomical structure, a more stable blood flow can be maintained. As reported, orthotopic auxiliary liver transplantation may lead to fewer complications than heterotopic transplantation (18). Therefore, transplant surgeons usually make more effort to dissect adhesions rather than change the position of a graft. However, when we rolled the left lobe over, it was well matched with the right lobe in regard to the portal and hepatic veins. The risks of angulation, compression and twist can also be reduced by precise vessel reconstruction. No vessel graft was used in this case. In an APOLT for non-cirrhotic liver metabolic disease, a reduced left lobe graft blood supply was found (18). The relatively high blood flow resistance due to small volume, immunological, and ischemic injuries may result in a continuing reduction of the blood supply of the left graft, especially when in competition with the non-cirrhotic native liver. Placing the left lobe in the right side may contribute to the balance of the PV blood distribution because of the similar volumes of the donor and remnant native livers. More blood supply to a donor from the right branch of the PV may balance the risk of immune and ischemia injuries. Thus, we believe that this technique may have a special advantage in APOLTs for noncirrhotic liver diseases.

No complications of the blood vessels were found in any of the recipients. Anastomoses of the PVs were carefully designed in these cases. In patient $\mathrm{C}$, the left $\mathrm{PV}$ of the graft was reconstructed by the end to side method and the angulation of end to end anastomosis was avoided. The anastomosis of the PV was pushed to the opposite direction of the graft with the increasing volume of the graft after transplantation. This change should be taken into consideration before PV reconstruction. When the end of the donor's (left or right) PV is lower than the bifurcation of the recipient's PV, the end to side method was suggested. Biliary anastomotic strictures were found in the two cases that received end to end biliary anastomosis. Though an improvement was found after PTCD, choledochojejunostomy seemed to be an optimal way to prevent or treat this problem. All patients recovered and had a normal liver function.

As reported $(6,7,19)$ previously, current practice also supports auxiliary liver transplantation as an aid for a SFSS graft. However, in patients with severe liver cirrhosis, the function and PV blood flow of the liver decline to a large degree and a relatively large graft would be needed even for auxiliary liver transplantation. In these patients, hepatic failure, PV hypertension, and blood coagulation disorders may also increase the risk and complications of APOLT. Therefore, only patients with relatively mild cirrhosis and an indication of a gastrointestinal hemorrhage were considered for APOLT in the current series. Right lobe and whole liver grafts would be preferred for patents with more severe cirrhosis.

\section{CONCLUSION}

It is possible to block the transmission of HBV between the native liver and graft by a sensitive nucleos(t)ide drug treatment. Auxiliary transplantation of left lobe grafts may be a practical option for recipients at risk for SFSS. Left lobe grafts can also be implanted into the space of the right lobe of the liver safely.

\section{DATA AVAILABILITY STATEMENT}

The original contributions presented in the study are included in the article/supplementary material, further inquiries can be directed to the corresponding author/s.

\section{ETHICS STATEMENT}

The studies involving human participants were reviewed and approved by the ethics committee of Beijing Friendship Hospital. The patients/participants provided their written informed consent to participate in this study.

\section{AUTHOR CONTRIBUTIONS}

L-YS: participated in the research design and the patient management. Z-JZ: planned and performed the operations and participated in operations. JX and YL: participated in the patient management after the operations. WQ: participated in operations. C-DW: contributed to treatments and operations as an expert consultant. H-MZ: participated in data analysis and the writing of the paper. LW: participated in the research design and the surgical process of operations. All authors contributed to the article and approved the submitted version.

\section{FUNDING}

This work was supported by Capital's Funds for Health Improvement and Research (No. 2020-1-2024) and Beijing Municipal Science and Technology Commission (No. Z181100001718220). 


\section{REFERENCES}

1. Zhu ZJ, Wei L, Qu W, Sun LY, Liu Y, Zeng ZG, et al. First case of crossauxiliary double domino donor liver transplantation. World J Gastroenterol. (2017) 23:7939-44. doi: 10.3748/wjg.v23.i44.7939

2. Qu W, Wei L, Zhu ZJ, Sun LY, Liu Y, Zeng ZG. Considerations for use of domino cross-auxiliary liver transplantation in metabolic liver diseases: a review of case studies. Transplantation. (2019) 103:191620. doi: 10.1097/TP.0000000000002602

3. European Association for the Study of the Liver. Electronic address EEE. EASL clinical practice guidelines: liver transplantation. J Hepatol. (2016). 64:433-85. doi: 10.1016/j.jhep.2015.10.006

4. Fagiuoli S, Daina E, D’Antiga L, Colledan M, Remuzzi G. Monogenic diseases that can be cured by liver transplantation. J Hepatol. (2013) 59:595612. doi: 10.1016/j.jhep.2013.04.004

5. Jiang YZ, Sun LY, Zhu ZJ, Wei L, Qu W, Zeng ZG, et al. Perioperative characteristics and management of liver transplantation for isolated methylmalonic acidemia-the largest experience in China. Hepatobiliary Surg Nutr. (2019) 8:470-9. doi: 10.21037/hbsn.2019.03.04

6. Inomata Y, Kiuchi T, Kim I, Uemoto S, Egawa H, Asonuma K, et al. Auxiliary partial orthotopic living donor liver transplantation as an aid for small-for-size grafts in larger recipients. Transplantation. (1999) 67:13149. doi: 10.1097/00007890-199905270-00004

7. Kobayashi T, Sato Y, Yamamoto S, Oya H, Hara Y, Watanabe T, et al. Feasibility of auxiliary partial living donor liver transplantation for fulminant hepatic failure as an aid for small-for-size graft: single center experience. Transplant Proc. (2009) 41:262-4. doi: 10.1016/j.transproceed.2008.10.025

8. Terpstra OT, Schalm SW, Weimar W, Willemse PJ, Baumgartner $\mathrm{D}$, Groenland $\mathrm{TH}$, et al. Auxiliary partial liver transplantation for end-stage chronic liver disease. $N$ Engl J Med. (1988) 319:1507-11. doi: 10.1056/NEJM198812083192303

9. ten Kate FJ, Schalm SW, Willemse PJ, Blok AP, Heijtink RA, Terpstra OT. Course of hepatitis B and D virus infection in auxiliary liver grafts in hepatitis B-positive patients. A light-microscopic and immunohistochemical study. $J$ Hepatol. (1992). 14:168-75. doi: 10.1016/0168-8278(92)90154-H

10. ten Kate FJ, Schalm SW. Course of hepatitis delta virus infection in auxiliary liver grafts in patients with delta virus cirrhosis. Prog Clin Biol Res. (1991). 364:429-37.

11. Durand F, Belghiti J, Handra-Luca A, Francoz C, Sauvanet A, Marcellin P, et al. Auxiliary liver transplantation for fulminant hepatitis B: results from a series of six patients with special emphasis on regeneration and recurrence of hepatitis B. Liver Transpl. (2002) 8:701-7. doi: 10.1053/jlts.2002.33745

12. Quaglia A, Portmann BC, Knisely AS, Srinivasan P, Muiesan P, Wendon J, et al. Auxiliary transplantation for acute liver failure: histopathological study of native liver regeneration. Liver Transpl. (2008) 14:1437-48. doi: 10.1002/lt.21568
13. Wang SF, Chen XP, Chen ZS, Wei L, Dong SL, Guo H, et al. Left lobe auxiliary liver transplantation for end-stage hepatitis B liver cirrhosis. Am J Transplant. (2017) 17:1606-12. doi: 10.1111/ajt.14143

14. Uchiyama H. Prudent use of a left hepatic graft in adult-to-adult living donor liver transplantation: aiming for further securing donors' safety. Hepatobiliary Surg Nutr. (2020) 9:360-3. doi: 10.21037/hbsn.2019.11.04

15. Wang $\mathrm{P}$, Tam $\mathrm{N}$, Wang $\mathrm{H}$, Zheng $\mathrm{H}$, Chen $\mathrm{P}$, Wu $\mathrm{L}$, et al. Is hepatitis B immunoglobulin necessary in prophylaxis of hepatitis B recurrence after liver transplantation? A meta-analysis. PLoS ONE. (2014) 9:e104480. doi: 10.1371/journal.pone.0104480

16. Cholongitas E, Papatheodoridis GV. High genetic barrier nucleos(t)ide analogue(s) for prophylaxis from hepatitis B virus recurrence after liver transplantation: a systematic review. Am J Transplant. (2013) 13:35362. doi: 10.1111/j.1600-6143.2012.04315.x

17. Fernandez I, Loinaz C, Hernandez O, Abradelo M, Manrique A, Calvo J, et al. Tenofovir/entecavir monotherapy after hepatitis B immunoglobulin withdrawal is safe and effective in the prevention of hepatitis B in liver transplant recipients. Transpl Infect Dis. (2015) 17:695-701. doi: 10.1111/tid.12434

18. Kasahara M, Takada Y, Kozaki K, Uryuhara K, Ogura Y, Ogawa K, et al. Functional portal flow competition after auxiliary partial orthotopic living donor liver transplantation in noncirrhotic metabolic liver disease. J Pediatr Surg. (2004) 39:1138-41. doi: 10.1016/j.jpedsurg.2004.03.079

19. Kasahara M, Kiuchi T, Uryuhara K, Takakura K, Egawa H, Asonuma K, et al. Auxiliary partial orthotopic liver transplantation as a rescue for small-forsize grafts harvested from living donors. Transplant Proc. (1998) 30:1323. doi: 10.1016/S0041-1345(97)01210-4

Conflict of Interest: The authors declare that the research was conducted in the absence of any commercial or financial relationships that could be construed as a potential conflict of interest.

Publisher's Note: All claims expressed in this article are solely those of the authors and do not necessarily represent those of their affiliated organizations, or those of the publisher, the editors and the reviewers. Any product that may be evaluated in this article, or claim that may be made by its manufacturer, is not guaranteed or endorsed by the publisher.

Copyright (C) 2021 Wei, Zhang, Wan, Qu, Zeng, Liu, Xiong, Sun and Zhu. This is an open-access article distributed under the terms of the Creative Commons Attribution License (CC BY). The use, distribution or reproduction in other forums is permitted, provided the original author(s) and the copyright owner(s) are credited and that the original publication in this journal is cited, in accordance with accepted academic practice. No use, distribution or reproduction is permitted which does not comply with these terms. 